Check for updates

1 Watford, UK

2 Imperial College London, UK

a.majeed@imperial.ac.uk Follow Simon Hodes on Twitter @DrSimonHodes Follow Azeem Majeed on Twitter @Azeem_Majeed

Cite this as: $B M J 2021 ; 373: n 1578$ http://dx.doi.org/10.1136/bmj.n1578 Published: 21 June 2021

\title{
Building a sustainable infrastructure for covid-19 vaccinations long term
}

\section{With covid-19 booster vaccinations likely in the near future, now is the time to consider how to create a sustainable, long term infrastructure for vaccine delivery}

\section{Simon Hodes, ${ }^{1}$ Azeem Majeed ${ }^{2}$}

By mid-June 2021, the UK had administered over 70 million doses of covid-19 vaccine, with around $75 \%$ delivered by primary care led vaccination sites. Since the start of the programme in December 2020, the UK has offered a variety of locations for vaccination: GP led sites, mass vaccine sites, community pharmacies, and hospitals. The vaccine rollout is a major and much needed success for the NHS, and there are many positive lessons to be learnt. We must not, however, be complacent. We are still in the midst of a global pandemic, with covid-19 rife in many countries and new, more infectious variants continually emerging. It is essential for the UK to maintain its vaccination momentum, as well as consider extending the programme to older children, and be prepared to offer booster doses to adults if these are required to maintain immunity. We must also focus on vaccine hesitancy, which is a major global health risk.

General practices in the UK are experienced at mass vaccination, being largely responsible for administering seasonal flu vaccines with support from community pharmacy sites. In 2020, the cohorts offered flu vaccines were extended to include household members of high risk patients, and all people aged 50-64. The same extended groups will be targeted for a flu vaccine next winter. General practices are embedded in their communities; are local and trusted; have health compliant regulated premises, rigorous cold storage systems, resuscitation equipment on site; hold full electronic patient records; and have longstanding knowledge of their patients. For all these reasons, when covid-19 vaccines became available, it was primary care teams who were able quickly to step forward and deliver most vaccinations.

The initial cohort for the covid vaccination programme-people aged 80 and over-were not easily reached by email or text messages, are sometimes not technically literate, and many needed phone calls to book their vaccination appointments. The amount of time and effort this took was considerable and it is a credit to overstretched primary care teams nationwide (with special praise for practice managers, receptionists, care coordinators, link workers, nurses, volunteers, and other support staff) that our most vulnerable patients, including nursing home, housebound, and clinically extremely vulnerable patients, received their initial injections so quickly and efficiently.

The logistics around the storage and handling of the Pfizer BioNTech vaccine precluded the use of community pharmacy sites, but the Oxford AstraZeneca (like the seasonal flu vaccine) has been widely administered at community sites. Recent changes by the Medicines and Healthcare Products Regulatory Agency allowing up to 31 days' storage in a vaccine fridge for the Pfizer vaccine has the potential to further expand the range of suitable sites for its use.

In England, GPs have been working in primary care networks (PCNs), representing groups of practices typically covering 30000 to 50 ooo patients, since July 2019. PCNs are ideally placed to offer population based health services including covid-19 vaccination. Some PCNs have offered covid-19 vaccinations in-house by reorganising their services, while others have worked with other PCNs to use large sites such as sports centres, entertainment venues, and village halls. Many PCNs have offered "pop-up clinics"-for example in homeless shelters, community centres, places of worship, and hostels-with great success. These pop-up clinics have increased uptake in marginalised groups who are typically at higher risk of infection, serious illness, and complications from covid-19, thereby helping to reduce health inequalities.

There has been great commitment from both the existing primary care workforce and volunteers to deliver the vaccination programme, with many retired staff coming back to help, primary care staff being redeployed, and volunteers acting in roles such as marshals, data entry clerks, and car park wardens. In addition to the administrative workload, there are huge numbers of queries from patients about their vaccines, both before and after the event. This hidden work is also being carried out, unfunded and largely unrecognised, by primary care teams. Each time there is a change in policy by the government or a health scare in the media, primary care teams are inundated with calls, and this workload and its importance in ensuring the continued high uptake of covid-19 vaccines needs to be recognised by the government.

The UK government has recently announced that it may offer a booster in autumn 2021 and, with new variants emerging, this is likely to be a key health policy to protect the NHS over the winter. ${ }^{1}$ Hence, now is the right time to appraise the options for vaccine delivery. Having a mixed range of sites able to offer mass vaccination should, in theory, speed up the process, but it has been clear that the limiting factor is vaccine supply, not capacity to vaccinate. ${ }^{2}$

We recommend NHS England publish data on the respective costs of delivering vaccines through PCNs 
(general practice sites) compared with mass vaccine centres. Any such calculations must include set up and running costs, and also explain where the clinical staff are coming from-knowing that staff shortages are already over $10 \%$ across the NHS. ${ }^{3}$ The work in dealing with queries from patients also needs to be factored into this evaluation-this is currently largely being directed at primary care teams, who are often left to deal with the most complex patients. We would also request an official breakdown of the percentage of vaccines given in each setting thus far, so that there is full transparency, as well as an appreciation for the huge efforts made by GP teams.

By using primary care sites for the vaccination programme, there is the potential to invest in and strengthen our infrastructure for local healthcare delivery, which will assist in NHS recovery plans and leave a legacy. By contrast, there is a risk that mass vaccine sites-like the Nightingale hospitals-will eventually be dismantled. Policy makers need carefully to evaluate the use of mass vaccine sites and GP led sites, along with the desire of patients to receive their care closer to home and in a familiar setting. There must be adequate resources for this work, so that routine care and timely access to other primary care services is not compromised.

We know that many GP teams are at breaking point and must be fully supported if they are to provide mass covid-19 vaccination in addition to their core work. Investment in primary care led vaccination sites, supported by local pharmacies, is likely to be the most cost effective option for ongoing mass vaccination, as well as being the option that is preferred by most patients. It is essential, therefore, for the government and NHS managers to work with primary care teams, giving them the resources needed to put in place a sustainable, long term infrastructure for vaccine delivery.

Competing interests: none declared.

Not commissioned, not peer reviewed.

AM is supported by the NIHR Applied Research Collaboration NW London. The views expressed are those of the authors and not necessarily those of the NHS, the NIHR, or the Department of Health and Social Care.

1 Mahase E. Covid-19: Booster vaccine to be rolled out in autumn as UK secures 60m more Pfizer doses. BMJ 2021;373:n1116. doi: 10.1136/bmj.n1116 pmid: 33926905

2 All UK adults could get covid vaccine dose by June if supply speeds up. Guardian March 2021. www.theguardian.com/society/2021/mar/03/all-uk-adults-could-get-covid-vaccine-dose-by-juneif-supply-speeds-up

3 Oliver D. David Oliver: Do the NHS workforce plans really add up?BMJ2020;370:m3203. doi: 10.1136/bmj.m3203 pmid: 32819892

This article is made freely available for use in accordance with BMJ's website terms and conditions for the duration of the covid-19 pandemic or until otherwise determined by BMJ. You may use, download and print the article for any lawful, non-commercial purpose (including text and data mining) provided that all copyright notices and trade marks are retained. 